\title{
COMMENTS ON DING'S EXAMPLES OF $F C$-SPACES AND RELATED MATTERS
}

\author{
SEHIE PARK
}

\begin{abstract}
Recently Ding $[4,5,8]$ gives examples of his $F C$-spaces which are not $L$-spaces due to Ben-El-Mechaiekh et al. [1]. We show that they are actually $L$-spaces. We also clarify that all statements in [5] can be stated in corrected and generalized forms for the class of abstract convex spaces beyond $F C$-spaces.
\end{abstract}

\section{Introduction}

In a sequence of more than a dozen papers, X. P. Ding introduced the socalled $F C$-spaces and claimed that his spaces contain the classes of $L$-spaces and $G$-convex spaces. In our previous works $[14,19]$ and a number of other papers, it is shown that Ding's $F C$-spaces are $L$-spaces due to Ben-El-Mechaiekh et al. [1] and hence $G$-convex spaces, on the contrary to Ding's claim; see the references of [19]. Ding's misconception is mainly based on his incautious reading of [1] where its authors did not claim that their $L$-spaces (which are misquoted as $L$-convex spaces in Ding's papers) generalize $G$-convex spaces. Ding's misconception appeared too many papers of his own and of many followers, and this was already clarified in [14, 19] and some subsequent papers. Recently, Ding gave false examples of $F C$-spaces which are not $L$-spaces as [4, Example 1.1], [5, Examples 2.1 and 2.2], and [8, Example 2.1]. Even in 2009, such misconceptions have appeared successively in other papers of Ding $[6,7]$ and his followers $[2,3]$. Our first aim in the present paper is to destroy such misconceptions.

Moreover, in [5], Ding deals with the so-called generalized $R-K K M$ maps on $F C$-spaces, which are very particular to $K K M$-maps on abstract convex spaces due to the present author [15-18]. By using an $R-K K M$ type theorem in $F C$ spaces, Ding [5] proves some minimax inequalities involving two bifunctions with noncompact and nonconvex domains in $F C$-spaces. As applications, he

Received August 24, 2010.

2010 Mathematics Subject Classification. 47H04, 47H10, 49J27, 49J35, 54C60, 54H25, 91B50.

Key words and phrases. abstract convex space, KKM space, generalized $(G-)$ convex space, $L$-space, minimax inequality, fixed point. 
obtained some Fan-Browder type fixed point theorems for expansive multimaps with noncompact and nonconvex domains and ranges in general topological spaces. Moreover, in [5], in order to state utmost generally, its author adopts terminology like compactly open (or closed) sets, compactly open neighborhood, transfer lower semicontinuous functions, transfer compactly open (or closed) sets, and transfer compactly lower semicontinuous maps. It is already wellknown that, by replacing the original topology by its compactly generated extension, we can easily eliminate the "compactly" concepts throughout [5] and a large number of other papers; see [12].

Recall that a multimap $F: X \multimap Y$ from a set $X$ to a topological space $Y$ has transfer closed values whenever $\bigcap_{x \in X} G(x)=\bigcap_{x \in X} \overline{G(x)}$; and transfer open values whenever $\bigcup_{x \in X} G(x)=\bigcup_{x \in X} \operatorname{Int} G(x)$. Therefore, the use of the transfer terminology is not much general than one expects, and we can obtain the "transfer" version easily from the ordinary statements if necessary; see [12]. Consequently, statements adopting transfer open (or closed) sets are equivalent to corresponding statements adopting mere open (or closed) sets.

In the present paper, we show that Ding's examples of $F C$-spaces in $[5,8]$ are actually $L$-spaces due to Ben-El-Mechaiekh et al. [1]. We also clarify that all statements in [5] can be stated in corrected and generalized forms for the class of abstract convex spaces beyond $F C$-spaces.

\section{Preliminaries}

Multimaps are also called simply maps. Let $\langle D\rangle$ denote the set of all nonempty finite subsets of a set $D$. Recall the following in [15-18, 21]:

Definition. An abstract convex space $(E, D ; \Gamma)$ consists of a topological space $E$, a nonempty set $D$, and a multimap $\Gamma:\langle D\rangle \rightarrow 2^{E}$ with nonempty values $\Gamma_{A}:=\Gamma(A)$ for $A \in\langle D\rangle$.

For any $D^{\prime} \subset D$, the $\Gamma$-convex hull of $D^{\prime}$ is denoted and defined by

$$
\operatorname{co}_{\Gamma} D^{\prime}:=\bigcup\left\{\Gamma_{A} \mid A \in\left\langle D^{\prime}\right\rangle\right\} \subset E .
$$

A subset $X$ of $E$ is called a $\Gamma$-convex subset of $(E, D ; \Gamma)$ relative to $D^{\prime}$ if for any $N \in\left\langle D^{\prime}\right\rangle$, we have $\Gamma_{N} \subset X$, that is, $\operatorname{co}_{\Gamma} D^{\prime} \subset X$. Then $\left(X, D^{\prime} ;\left.\Gamma\right|_{\left\langle D^{\prime}\right\rangle}\right)$ is called a $\Gamma$-convex subspace of $(E, D ; \Gamma)$.

When $D \subset E$, the space is denoted by $(E \supset D ; \Gamma)$. In such case, a subset $X$ of $E$ is said to be $\Gamma$-convex if $\operatorname{co}_{\Gamma}(X \cap D) \subset X$; in other words, $X$ is $\Gamma$-convex relative to $D^{\prime}:=X \cap D$. In case $E=D$, let $(E ; \Gamma):=(E, E ; \Gamma)$.

Example. There are plenty of examples of abstract convex spaces; see [15-18, 21]. Here we need only two subclasses:

(1) A generalized convex space or a $G$-convex space $(X, D ; \Gamma)$ due to Park is an abstract convex space such that for each $A \in\langle D\rangle$ with the cardinality $|A|=n+1$, there exists a continuous function $\phi_{A}: \Delta_{n} \rightarrow \Gamma(A)$ such that $J \in\langle A\rangle$ implies $\phi_{A}\left(\Delta_{J}\right) \subset \Gamma(J)$. 
Here, $\Delta_{n}$ is the standard $n$-simplex with vertices $\left\{e_{i}\right\}_{i=0}^{n}, \Delta_{J}$ its face corresponding to $J \in\langle A\rangle$; that is, if $A=\left\{a_{0}, a_{1}, \ldots, a_{n}\right\}$ and $J=\left\{a_{i_{0}}, a_{i_{1}}, \ldots, a_{i_{k}}\right\}$ $\subset A$, then $\Delta_{J}=\operatorname{co}\left\{e_{i_{0}}, e_{i_{1}}, \ldots, e_{i_{k}}\right\}$.

A $G$-convex space $(X ; \Gamma)$ is called an $L$-space by Ben-El-Mechaiekh et al. $[1]$.

(2) A $\phi_{A^{-}}$space $\left(X, D ;\left\{\phi_{A}\right\}_{A \in\langle D\rangle}\right)$ consists of a topological space $X$, a nonempty set $D$, and a family of continuous functions $\phi_{A}: \Delta_{n} \rightarrow X$ (that is, singular $n$-simplexes) for $A \in\langle D\rangle$ with $|A|=n+1$. Every $\phi_{A}$-space can be made into a $G$-convex space; see $[14,19]$. The so-called $F C$-spaces $\left(X ;\left\{\varphi_{A}\right\}\right)$ defined by Ding are particular type of $\phi_{A}$-spaces for the case $X=D$. Later, $\phi_{A}$-spaces are called GFC-spaces in [11].

We have the following:

Theorem 1. Any FC-space $\left(X ;\left\{\varphi_{A}\right\}\right)$ can be made into an $L$-space $(X ; \Gamma)$.

Proof. This can be done at least in three ways.

(1) For each $A \in\langle X\rangle$, by putting $\Gamma_{A}:=X$, we obtain a trivial $L$-space $(X ; \Gamma)$.

(2) Let $\left\{\Gamma^{\alpha}\right\}_{\alpha}$ be the family of maps $\Gamma^{\alpha}:\langle X\rangle \rightarrow 2^{X}$ giving an $L$-space $\left(X ; \Gamma^{\alpha}\right)$. Note that, by (1), this family is not empty. Then, for each $\alpha$ and each $A \in\langle X\rangle$ with $|A|=n+1$, we have

$$
\varphi_{A}\left(\Delta_{n}\right) \subset \Gamma_{A}^{\alpha} \text { and } \varphi_{A}\left(\Delta_{J}\right) \subset \Gamma_{J}^{\alpha} \text { for } J \subset A \text {. }
$$

Let $\Gamma:=\bigcap_{\alpha} \Gamma^{\alpha}$, that is, $\Gamma_{A}=\bigcap_{\alpha} \Gamma_{A}^{\alpha}$. Then

$$
\varphi_{A}\left(\Delta_{n}\right) \subset \Gamma_{A} \text { and } \varphi_{A}\left(\Delta_{J}\right) \subset \Gamma_{J} \text { for } J \subset A \text {. }
$$

Therefore, $(X ; \Gamma)$ is an $L$-space.

(3) Let $N \in\langle X\rangle$ with $|N|=n+1$. For each $M \in\langle X\rangle$ with $N \subset M$, $M=\left\{a_{0}, \ldots, a_{m}\right\}$ and $N=\left\{a_{i_{0}}, \ldots, a_{i_{n}}\right\}$, there exists a subset $\varphi_{M}\left(\Delta_{n}^{M}\right)$ of $X$ such that $\Delta_{n}^{M}:=\operatorname{co}\left\{e_{i_{j}} \mid j=0, \ldots, n\right\} \subset \Delta_{m}$. Now let

$$
\Gamma_{N}=\Gamma(N):=\bigcup_{M \supset N} \varphi_{M}\left(\Delta_{n}^{M}\right) .
$$

Then $\Gamma:\langle X\rangle \rightarrow 2^{X}$ is well-defined and $(X ; \Gamma)$ becomes an $L$-space: In fact, for each $A \in\langle X\rangle$ with $|A|=n+1$, there exists a continuous map $\varphi_{A}: \Delta_{n} \rightarrow \Gamma(A)$ such that $J \in\langle A\rangle$ implies $\varphi_{A}\left(\Delta_{J}\right) \subset \Gamma(J)$.

\section{Ding's examples}

In many of his previous works and even in 2009 [5-8], Ding repeatedly stated that his class of $F C$-spaces includes that of $G$-convex spaces among others. One wonders how could a pair $\left(X ;\left\{\varphi_{A}\right\}\right)$ generalize a triple $(X, D ; \Gamma)$. In $[4,5,8]$, he also gives several fake examples of $F C$-spaces which are not $L$-spaces. In fact, he merely shows that given $\varphi_{N}\left(\Delta_{n}\right)$ can not be $\Gamma(N)$ in $L$-spaces and fails to show no existence of $L$-structure in given spaces.

He stated repeatedly as follows, for example, in [6]: 
"By comparing the definitions, it is easy to see that any convex subset of a topological vector space, any $H$-space introduced by Horvath, any $G$-convex space introduced by Park and Kim, and any $L$-convex spaces introduced by Ben-El-Mechaiekh et al. [1] are all $F C$-space. Some examples of $F C$-spaces which are not $L$-convex spaces have been given by the Example 1.1 of Ding [4] and Examples 2.1 and 2.2 of Ding [5]. Hence, it is quite reasonable and valuable to study various nonlinear problems in $F C$-spaces."

This statement is incorrect by the following reasons:

(1) Recall that the $G$-convex space $(X, D ; \Gamma)$ is originated from the original KKM theorem and the celebrated Ky Fan lemma from the beginning, where $X \neq D$. The case $X=D$ is not applicable to them and this is the most serious defect of $L$-spaces or $F C$-spaces since they are inadequate for the KKM theory.

(2) By defining $\Gamma(N)=X$ for all $N \in\langle X\rangle$, Ding's $F C$-space becomes trivially an $L$-space, and hence a $G$-convex space.

(3) We gave already at least two more proofs showing that any $F C$-space can be made into an $L$-space; see Theorem 1.

(4) In Ding's new examples, he has to show that any $\Gamma$ does not work, not a particular one. Moreover, his $\Gamma$ is not well chosen. There exists a suitable $\Gamma$ (even not the one in Case (1) of Theorem 1) such that his claim is false. See below:

Example 2.1 ([5]). Let $X_{1}$ and $X_{2}$ be two nonempty convex subsets of a topological vector space $X$ with $\operatorname{cl} X_{1} \cap \operatorname{cl} X_{2}=\emptyset$ and $g: X_{2} \rightarrow X_{1}$ be a single-valued mapping. Then $E=X_{1} \cup X_{2}$ is not convex. For each $N=$ $\left\{x_{0}, \ldots, x_{n}\right\} \in\langle E\rangle$, define a mapping $\varphi_{N}: \Delta_{n} \rightarrow 2^{X}$ by

$$
\varphi_{N}(\alpha)=\left\{\begin{array}{cl}
\sum_{i=0}^{n} \alpha_{i} x_{i} & \text { if } N \subset X_{1} \text { or } N \subset X_{2} ; \\
\sum_{i=0}^{j} \alpha_{i} x_{i}+\sum_{i=j+1}^{n} \alpha_{i} g\left(x_{i}\right) & \text { if } N=N_{1} \cup N_{2}
\end{array}\right.
$$

for all $\alpha=\left(\alpha_{0}, \ldots, \alpha_{n}\right) \in \Delta_{n}$ where $N_{1}=\left\{x_{0}, \ldots, x_{j}\right\} \subset X_{1}, N_{2}=\left\{x_{j+1}, \ldots\right.$, $\left.x_{n}\right\} \subset X_{2}$. It is easy to see that $\varphi_{N}$ is continuous and hence $\left(E, \varphi_{N}\right)$ is an $F C$-space.

If we define a set-valued mapping $\Gamma:\langle E\rangle \rightarrow 2^{E}$ by

$$
\Gamma(N)=\varphi_{N}\left(\Delta_{n}\right), \forall N=\left\{x_{0}, \ldots, x_{n}\right\} \in\langle E\rangle,
$$

then we have that for each $N=\left\{x_{0}, \ldots, x_{n}\right\} \in\langle E\rangle, \varphi_{N}\left(\Delta_{n}\right) \subset \Gamma(N)$. But if $N=N_{1} \cup N_{2}$ where $N_{1}=\left\{x_{0}, \ldots, x_{j}\right\} \subset X_{1}$ and $N_{2}=\left\{x_{j+1}, \ldots, x_{n}\right\} \subset$ $X_{2}$, then we have $\Gamma\left(N_{2}\right)=\varphi_{N_{2}}\left(\Delta_{J}\right) \subset X_{2}$ and $\varphi_{N}\left(\Delta_{J}\right) \subset X_{1}$, where $\Delta_{J}=$ $\operatorname{co}\left\{e_{k} \mid k=j+1, \ldots, n\right\}$. Hence we have $\varphi_{N}\left(\Delta_{J}\right) \not \subset \Gamma\left(N_{2}\right)$. Hence $(E, \Gamma)$ is not an $L$-convex space. 
Comments. In this example, Ding showed that $\left(E, \varphi_{N}\right)$ is an $F C$-space, and $(E, \Gamma)$ with the particular $\Gamma(N)=\varphi_{N}\left(\Delta_{n}\right)$ is not an $L$-space. Yes, this particular $\Gamma$ does not work. But he should show that any $\Gamma$ does not work.

In fact, if we define a multimap $\Gamma:\langle E\rangle \rightarrow 2^{E}$ by

$$
\Gamma(N)=\left\{\begin{array}{cl}
\varphi_{N}\left(\Delta_{n}\right) \cup X_{1} & \text { if } N \subset X_{1} \text { or } N \subset X_{2} ; \\
\varphi_{N}\left(\Delta_{n}\right) & \text { if } N=N_{1} \cup N_{2},
\end{array}\right.
$$

where $N_{1}=\left\{x_{0}, \ldots, x_{j}\right\} \subset X_{1}, N_{2}=\left\{x_{j+1}, \ldots, x_{n}\right\} \subset X_{2}$, then it is easily checked that $\varphi_{N}\left(\Delta_{n}\right) \subset \Gamma(N)$ and $\varphi_{N}\left(\Delta_{J}\right) \subset \Gamma(J)$ for any $J \subset N$. Therefore $(X, \Gamma)$ becomes an $L$-space.

Example 2.2 ([5]). Let $(X,\|\cdot\|)$ be a strictly convex and reflexive Banach space and $X_{1}$ is a nonempty closed convex subset of $X$ and $X_{2}$ be a nonempty convex subset of $X$ with $X_{1} \cap X_{2}=\emptyset$. Then $E=X_{1} \cup X_{2}$ is not convex. For each $N=\left\{x_{0}, \ldots, x_{n}\right\} \in\langle E\rangle$, define a mapping $\varphi_{N}: \Delta_{n} \rightarrow 2^{X}$ as in Example 2.1 where $g: X_{2} \rightarrow X_{1}$ is replaced by the metric project mapping $P_{X_{1}}: X_{2} \rightarrow X_{1}$. Then $\left(E, \varphi_{N}\right)$ is an $F C$-space which is not an $L$-convex space.

Comments. Similarly to the preceding comments, Ding's claim is wrong.

Example 2.1 ([8]). Let $X=(1,2) \cup(3,+\infty)$ with usual topology. Define a mapping $\varphi_{N}: \Delta_{n} \rightarrow 2^{X}$ as follows: for each $N=\left\{x_{0}, \ldots, x_{n}\right\} \in\langle X\rangle$ and for each $\alpha=\left(\alpha_{0}, \ldots, \alpha_{n}\right) \in \Delta_{n}$,

$$
\varphi_{N}(\alpha)=\left\{\begin{array}{cl}
\sum_{i=0}^{n} \alpha_{i} x_{i} & \text { if } N \subset(1,2) ; \\
3 \sum_{i=0}^{n} \alpha_{i} x_{i} & \text { if } N \not \subset(1,2) .
\end{array}\right.
$$

It is clear that $\varphi_{N}$ is continuous and hence $\left(X, \varphi_{N}\right)$ is an $F C$-space.

If we define a set-valued mapping $\Gamma:\langle X\rangle \rightarrow 2^{X}$ by

$$
\Gamma(N)=\varphi_{N}\left(\Delta_{n}\right), \forall N=\left\{x_{0}, \ldots, x_{n}\right\} \in\langle X\rangle,
$$

then we have that for each $N=\left\{x_{0}, \ldots, x_{n}\right\} \in\langle X\rangle, \varphi_{N}\left(\Delta_{n}\right) \subset \Gamma(N)$. But if $N=N_{1} \cup N_{2}$ where $N_{1} \subset(1,2)$ with $\left|N_{1}\right|=J+1, J<n$ and $N_{2} \subset$ $(3,+\infty)$, then we have $\Gamma\left(N_{1}\right)=\varphi_{N_{1}}\left(\Delta_{J}\right) \subset(1,2)$ and $\varphi_{N}\left(\Delta_{J}\right) \subset(3,+\infty)$, i.e., $\varphi_{N}\left(\Delta_{J}\right) \not \subset \Gamma\left(N_{1}\right)$. Hence $(X, \Gamma)$ is not an $L$-convex space.

Comments. In this example, Ding defines

$$
\varphi_{N}\left(\Delta_{n}\right)=\left\{\begin{array}{cl}
{[\min N, \max N]} & \text { if } N \subset(1,2) ; \\
{[3 \min N, 3 \max N]} & \text { if } N \not \subset(1,2) .
\end{array}\right.
$$

In [8], he defines $\Gamma(N)=\varphi_{N}\left(\Delta_{n}\right)$ and shows that $(X, \Gamma)$ is not an $L$-space. This particular $\Gamma$ does not work. But he should show that any $\Gamma$ does not work.

We already mentioned that, by letting $\Gamma^{1}(N):=X$, then $\left(X, \Gamma^{1}\right)$ becomes an $L$-space. 
We have another $\Gamma$ which makes $(X, \Gamma)$ an $L$-space. Define

$$
\begin{array}{rlrl}
\Gamma^{2}(N) & =\varphi_{N}\left(\Delta_{n}\right) \cup[3 \min N, 3 \max N] & \text { if } & N \subset(1,2) ; \\
& =\varphi_{N}\left(\Delta_{n}\right) & \text { if } N \not \subset(1,2) .
\end{array}
$$

Then it is clear that $\varphi_{N}\left(\Delta_{n}\right) \subset \Gamma^{2}(N)$ and $\varphi_{N}\left(\Delta_{J}\right) \subset \Gamma^{2}(J)$ for any $J \subset N$. Therefore $\left(X, \Gamma^{2}\right)$ is an $L$-space.

Remarks. 1. An example similar to the preceding one is also given by Ding and Fang [10, Example 2.1]. In [9], Ding still repeats his incorrect claims.

2. All results in [5] are mere generalizations or modifications of known ones to $F C$-spaces and seem to be artificial. The author often claims that his results generalize other works. This is doubtful since $F C$-spaces are $L$-spaces and hence, have no proper example.

Moreover, Ding's use of "compactly" open [closed] sets does not generalize anything and is not practical. In fact, by replacing the original topology by its compactly generated extension, they become mere open [closed] sets. Recall that Ding misguided many naive readers or his followers for a long period by using compact closure ccl or compact interior cint, without giving any proper example (Recently he seems to be not using ccl and cint; see [5-8]. But some naive followers are still using them; see [2]).

\section{KKM theorems}

In order to discuss the contents of [5], we need the following:

Definition. Let $(E, D ; \Gamma)$ be an abstract convex space. If a map $G: D \rightarrow 2^{E}$ satisfies

$$
\Gamma_{A} \subset G(A):=\bigcup_{y \in A} G(y) \quad \text { for all } A \in\langle D\rangle,
$$

then $G$ is called a KKM map.

Example ([5, Definition 2.3]). Let $X$ be a nonempty set and $Y$ a topological space. A multimap $G: X \rightarrow 2^{Y}$ is called a generalized $R-K K M$ mapping if for any $A=\left\{x_{0}, x_{1}, \ldots, x_{n}\right\} \in\langle X\rangle$ there exists a continuous map $\varphi_{A}: \Delta_{n} \rightarrow Y$ such that for each $\left\{x_{i_{0}}, x_{i_{1}}, \ldots, x_{i_{k}}\right\} \subset A$, we have

$$
\varphi_{A}\left(\Delta_{k}\right) \subset \bigcup_{j=1}^{k} G\left(x_{i_{j}}\right),
$$

where $\Delta_{k}=\operatorname{co}\left\{e_{i_{0}}, e_{i_{1}}, \ldots, e_{i_{k}}\right\}$.

Let us point out that $G$ is a simply KKM map on an abstract convex space $(Y, X ; \Gamma)$, where $\Gamma_{A}:=\varphi_{A}\left(\Delta_{n}\right)$. For more details, see [20].

Definition. The partial KKM principle for an abstract convex space $(E, D ; \Gamma)$ is the statement that, for any closed-valued KKM map $G: D \multimap E$, the family $\{G(y)\}_{y \in D}$ has the finite intersection property. The KKM principle is the statement that the same property also holds for any open-valued KKM map. 
An abstract convex space is called a KKM space if it satisfies the KKM principle.

In our recent works $[15-18,21]$, we studied the elements or foundations of the KKM theory on abstract convex spaces and noticed there that many important results therein are related to the partial KKM principle.

Example. We give only three examples of KKM spaces:

(1) Every $G$-convex space is a KKM space.

(2) A connected linearly ordered space $(X, \leq)$ can be made into a KKM space.

(3) The extended long line $L^{*}$ is a KKM space $\left(L^{*}, D ; \Gamma\right)$ with the ordinal space $D:=[0, \Omega]$. But $L^{*}$ is not a $G$-convex space.

Moreover, from the partial KKM principle we have a whole intersection property of the Fan type as follows:

Theorem 2. Generalized partial KKM principle. Let $(E, D ; \Gamma)$ be an abstract convex space satisfying the partial KKM principle and $G: D \multimap E$ a map such that

(1) $G$ is closed-valued;

(2) $G$ is a KKM map (that is, $\Gamma_{A} \subset G(A)$ for all $A \in\langle D\rangle$ ); and

(3) there exists a nonempty compact subset $K$ of $E$ such that one of the following holds:

(i) $K=E$;

(ii) $K=\bigcap\{G(z) \mid z \in M\}$ for some $M \in\langle D\rangle$; or

(iii) for each $N \in\langle D\rangle$, there exists a compact $\Gamma$-convex subset $L_{N}$ of $E$ relative to some $D^{\prime} \subset D$ such that $N \subset D^{\prime}$ and

$$
L_{N} \cap \bigcap_{z \in D^{\prime}} G(z) \subset K .
$$

Then $K \cap \bigcap\{G(z) \mid z \in D\} \neq \emptyset$.

Proof. Case (i): In this case every $G(y)$ is compact. Hence Case (i) reduces to (ii).

Case (ii): Since $\{G(z) \mid z \in D\}$ has the finite intersection property, so does $\{K \cap G(z) \mid z \in D\}$ in the compact set $K$. Hence it has the whole intersection property.

Case (iii): Suppose that $K \cap \bigcap\{G(z) \mid z \in D\}=\emptyset$; that is, $K \subset \bigcup\{X \backslash$ $G(z) \mid z \in N\}$ for some $N \in\langle D\rangle$. Let $L_{N}$ be the compact $\Gamma$-convex subset of $E$ in (iii). Define $G^{\prime}: D^{\prime} \multimap L_{N}$ by $G^{\prime}(z):=G(z) \cap L_{N}$ for $z \in D^{\prime}$. Then $A \in\left\langle D^{\prime}\right\rangle$ implies $\Gamma_{A}^{\prime}:=\Gamma_{A} \cap L_{N} \subset G(A) \cap L_{N}=G^{\prime}(A)$ by $(2)$; and hence $G^{\prime}: D^{\prime} \multimap L_{N}$ is a KKM map on $\left(L_{N}, D^{\prime} ; \Gamma^{\prime}\right)$ with closed values. Since $(X, D ; \Gamma)$ satisfies the partial KKM principle, so does $\left(L_{N}, D^{\prime} ; \Gamma^{\prime}\right)$; see [15, Lemma 2]. Hence, 
$\left\{G^{\prime}(z) \mid z \in D^{\prime}\right\}$ has the finite intersection property. Since $L_{N}$ is compact, and $\bigcap\left\{G^{\prime}(z) \mid z \in D^{\prime}\right\} \neq \emptyset$ by Case (i). For any

$$
y \in \bigcap_{z \in D^{\prime}}\left\{G^{\prime}(z)\right\} \subset L_{N} \cap \bigcap_{z \in D^{\prime}} G(z) \subset K,
$$

we have $y \in K$ by (iii). However, since $y \in K \subset \bigcup\{X \backslash G(z) \mid z \in N\}$, we have $y \notin G(z)$ for some $z \in N \subset D^{\prime}$. This contradicts $y \in \bigcap\left\{G^{\prime}(z) \mid z \in D^{\prime}\right\}$.

Therefore, we must have $K \cap \bigcap\{G(z) \mid z \in D\} \neq \emptyset$.

Theorem 2 appears in [21] and somewhere else, but we give its proof for completeness. Recall that conditions (i)-(iii) in Theorem 2 are usually called the compactness conditions or the coercivity conditions.

From Theorem 2, we can deduce an equivalent form of [17, Theorem 8.2]:

Corollary 2.1. Let $(X, D ; \Gamma)$ be an abstract convex space satisfying the partial $K K M$ principle, and $G: D \multimap X$ a map such that

(1) $\bigcap_{z \in D} G(z)=\bigcap_{z \in D} \overline{G(z)}$ [that is, $G$ is transfer closed-valued];

(2) $\bar{G}$ is a KKM map; and

(3) there exists a nonempty compact subset $K$ of $X$ such that one of the following holds:

(i) $K=X$;

(ii) $\bigcap\{\overline{G(z)} \mid z \in M\} \subset K$ for some $M \in\langle D\rangle$; or

(iii) for each $N \in\langle D\rangle$, there exists a compact $\Gamma$-convex subset $L_{N}$ of $X$ relative to some $D^{\prime} \subset D$ such that $N \subset D^{\prime}$ and

$$
L_{N} \cap \bigcap\left\{\overline{G(z)} \mid z \in D^{\prime}\right\} \subset K .
$$

Then $K \cap \bigcap\{G(z) \mid z \in D\} \neq \emptyset$.

Actually, Corollary 2.1 is equivalent to Theorem 2:

Proof. (Theorem $2 \Rightarrow$ Corollary 2.1) By putting $\overline{G(z)}$ instead of $G(z)$ in Theorem 2 for all $z \in D$, all of the requirements of Theorem 2 are satisfied. Then, by Theorem $2, K \cap \bigcap\{\overline{G(z)} \mid z \in D\} \neq \emptyset$. Since $G$ is transfer closed-valued by (1), we have the conclusion of Corollary 2.1.

(Corollary $2.1 \Rightarrow$ Theorem 2) Conversely, since $G(z)=\overline{G(z)}$ for all $z \in D$ in Theorem 2, all of the requirements of Corollary 2.1 are satisfied. Hence Theorem 2 holds.

Remarks. 1. From Theorem 2, we can deduce several equivalent formulations for abstract convex spaces satisfying the partial KKM principle as in [17].

2. In view of this equivalency, we do not need to think about the 'transfer' case. In fact, in Corollary 2.1, transfer closed sets can be replaced by closed sets. In this paper, we use only the closed-valued version and this will not loose any generality. 
Corollary 2.2 ([5, Theorem 3.1]). Let $X$ be a nonempty subset of an FC-space $\left(E, \varphi_{N}\right), Y$ be an $F C$-subspace of $E$ with $X \subset Y$ and $f, g: X \times Y \rightarrow \overline{\mathbb{R}}$ such that

(i) $f(x, y) \leq g(x, y)$ for all $(x, y) \in X \times Y$,

(ii) for each $N=\left\{x_{0}, \ldots, x_{n}\right\} \in\langle X\rangle$, each $\left\{x_{i_{0}}, \ldots, x_{i_{k}}\right\} \subset N$ and each $y \in \varphi\left(\Delta_{k}\right)$, there exists $j \in\{0, \ldots, k\}$ such that $g\left(x_{i_{j}}, y\right) \leq 0$,

(iii) there exists a nonempty compact subset $K$ of $Y$ such that for each $N \in$ $\langle X\rangle$, there exists a compact $F C$-space $L_{N}$ of $Y$ containing $N$ satisfying

$$
L_{N} \backslash K \subset \bigcup_{x \in X \cap L_{N}} \operatorname{int}_{Y}\{y \in Y \mid g(x, y)>0\} .
$$

Then $\bigcap_{x \in X} \operatorname{cl}_{Y}\{y \in Y \mid f(x, y) \leq 0\} \cap K \neq \emptyset$.

In addition, assume that

(iv) if $\{y \in Y \mid f(x, y) \leq 0\}$ is closed for each $x \in X$.

Then there exists $\hat{y} \in K$ such that $f(x, \hat{y}) \leq 0$ for all $x \in X$.

Proof. For each $N \in\langle X\rangle$, let $\Gamma_{N}=\varphi_{n}\left(\Delta_{n}\right)$ with $\varphi_{N}: \Delta_{n} \rightarrow Y$. Then $(Y \supset X ; \Gamma)$ is a KKM space since it is a $\phi_{A}$-space. Let $G(x)=\operatorname{cl}_{Y}\{y \in$ $Y \mid g(x, y) \leq 0\}$. By (ii), for each $N \in\langle X\rangle$ and $J \subset N$, we have

$$
\varphi_{N}\left(\Delta_{J}\right) \subset \bigcup_{x \in J}\{y \in Y \mid g(x, y) \leq 0\} \subset G(J) .
$$

Hence $G: X \multimap Y$ is a closed-valued KKM map.

Let $D^{\prime}=X \cap L_{N}$. Then, by (iii), we have

$$
L_{N} \cap \bigcap_{x \in D^{\prime}} \operatorname{cl}_{Y}\{y \in Y \mid g(x, y) \leq 0\}=L_{N} \cap \bigcap_{x \in D^{\prime}} G(x) \subset K .
$$

Therefore, by our KKM Theorem 2, we have $K \cap \bigcap_{x \in X} G(x) \neq \emptyset$. Since

$$
G(x)=\operatorname{cl}_{Y}\{y \in Y \mid g(x, y) \leq 0\} \subset \operatorname{cl}_{Y}\{y \in Y \mid g(x, y) \leq 0\},
$$

we have the first conclusion.

Further, if (iv) holds, from the first conclusion, we have

$$
\hat{y} \in K \cap \bigcap_{x \in X}\{y \in Y \mid f(x, y) \leq 0\} \neq \emptyset .
$$

This implies the second conclusion.

Remarks. 1. In [5, Theorem 3.1], instead of condition (iv), the following is assumed:

(iv) $)^{\prime} f(x, y)$ is transfer compactly lower semicontinuous.

This kind of requirement aims to seek utmost generality, but, is artificial, impractical, and useless. In fact, 'transfer' can be removed in view of the equivalency of Theorem 2 and Corollary 2.1; and 'compactly' can be destroyed by replacing the original topology of $Y$ by its compactly generated extension. Therefore, (iv)' simply tells nothing more than the following: 
(iv) $)^{\prime \prime} f(x, y)$ is lower semicontinuous.

In short, it is extremely difficult to give a concrete example of a function satisfying (iv)' but not (iv)".

2. Ding added a corollary [5, Corollary 3.1], noted that Włodarczyk and Klim [22, Propositions 4.1 and 4.2] follow from Corollary 2.2, and that [22, Propositions 4.3-4.5] can be also generalized.

3. In [5], from Corollary 2.2 [5, Theorem 3.1], Ding obtained two fixed point theorems [5, Theorems 4.1 and 4.2$]$ on $F C$-spaces.

\section{Fixed points of expansive multimaps on not necessarily convex or compact sets}

In this section, we begin new versions of results of Section 2 of $[13,22]$, and generalize [5, Theorem 4.3]:

Theorem 3. Let $C$ be a nonempty subset of an abstract convex space $(E ; \Gamma)$, $F, G: C \multimap E$, and $K$ a $\Gamma$-convex $K K M$ subspace of $(E ; \Gamma)$. Assume that the following conditions hold:

(1) for each $x \in C, G(x) \subset F(x)$;

(2) $C \subset K \subset G(C)$;

(3) for each $x \in C, G(x)$ is open [resp., closed] in $F(C)$;

(4) $G(C)=\bigcup_{i=1}^{n} G\left(c_{i}\right)$ for some $c_{1}, c_{2}, \ldots, c_{n} \in C$;

(5) for each $y \in K, F^{-}(y)$ is $\Gamma$-convex.

Then there exists $u \in C$ such that $u \in F(u)$.

Proof. Let $D:=\left\{c_{1}, c_{2}, \ldots, c_{n}\right\} \subset C$. Then $\left(K \supset D ;\left.\Gamma\right|_{\langle D\rangle}\right)$ is a KKM space. Let $T: D \multimap K$ be a map defined by $T\left(c_{i}\right)=K \backslash G\left(c_{i}\right)$ for each $i$. Then $T$ has closed [resp., open] values. Moreover, $\bigcap_{i=1}^{n} T\left(c_{i}\right)=K \backslash \bigcup_{i=1}^{n} G\left(c_{i}\right) \subset$ $G(C) \backslash \bigcup_{i=1}^{n} G\left(c_{i}\right)=\emptyset$. Therefore, by the KKM Theorem 2, $T$ can not be a KKM map. Hence, $\operatorname{co}_{\Gamma} N \not \subset T(N)$ for some $N \subset D$, that is, there exists a $u \in \operatorname{co}_{\Gamma} N \subset K$ such that $u \notin T\left(c_{j}\right)=K \backslash G\left(c_{j}\right)$ for each $c_{j} \in N$. Therefore $u \in G\left(c_{j}\right) \cap K$ or $c_{j} \in G^{-}(u) \subset F^{-}(u)$ for each $c_{j} \in N \subset C$. Since $F^{-}(u)$ is $\Gamma$-convex in $C$, we have $u \in \operatorname{co}_{\Gamma} N \subset F^{-}(u)$. Therefore, $u \in F(u)$. This completes our proof.

Remarks. 1. Note that [22, Theorem 2.1] is a particular form of the 'open' version of Theorem 3 with the additional requirement that (a) $E$ is Hausdorff, (b) $F(C)$ is a compact subset of $E$, and (c) $F^{-}(y)$ is nonempty. The authors of [22] adopted the partition of unity argument.

2. [13, Theorem 3.1] is the case of Theorem 3 for a t.v.s. $E$, a convex subset $K$ of $E$, and $F=G$. Similarly, other results in [13] can be generalized.

3. [5, Theorem 4.3] is the case of Theorem 3 where $(1)\left(K, \varphi_{N}\right)$ is an $F C$ space, (2) $G$ is transfer compactly open-valued, and (3) $G(X)$ is a compact subset of $E$. Note that our proof is different from that of [5, Theorem 4.3], where $G(X)$ should be Hausdorff. Ding used to miss this while he was using the partition of unity argument. 
4. From Theorem 3, as in [5], we can obtain generalized forms of [5, Corollaries 4.1-4.3] (which generalize results of [22]) and [5, Theorem 4.4].

\section{References}

[1] H. Ben-El-Mechaiekh, S. Chebbi, M. Florenzano, and J.-V. Llinares, Abstract convexity and fixed points, J. Math. Anal. Appl. 222 (1998), no. 1, 138-150.

[2] F.-P. Deng and L. Wang, Coincidence theorems in product FC-spaces, J. Sichuan Normal Univ. (Nat. Sci) 32 (2009), no. 3, 297-300.

[3] L. Deng and X.-Y. Zang, A parametric type of KKM theorem in FC-spaces with applications, Appl. Math. Mech. (English Ed.) 30 (2009), no. 1, 73-79.

[4] X. P. Ding, Maximal elements and generalized games involving condensing mappings in locally FC-uniform spaces and applications. I, Appl. Math. Mech. (English Ed.) 28 (2007), no. 12, 1561-1568.

[5] - Minimax inequalities and fixed points of expansive set-valued mappings with noncompact and nonconvex domains and ranges in topological spaces, Nonlinear Anal. 70 (2009), no. 2, 881-889.

[6] _ Pareto equilibria for generalized constrained multiobjective games in FC-spaces without local convexity structure, Nonlinear Anal. 71 (2009), no. 11, 5229-5237.

[7] _ Systems of generalized vector quasi-variational inclusions and systems of generalized vector quasi-optimization problems in locally FC-uniform spaces, Appl. Math. Mech. (English Ed.) 30 (2009), no. 3, 263-274.

[8] _ New systems of generalized vector quasi-equilibrium problems in product $F C$ spaces, J. Global Optim. 46 (2010), no. 1, 133-146.

[9] _ Collective fixed points, generalized games and systems of generalized quasivariational inclusion problems in topological spaces, Nonlinear Anal. 73 (2010), no. 6, 1834-1841.

[10] X. P. Ding and H. R. Feng, Fixed point theorems and existence of equilibrium points of noncompact abstract economies for $\mathcal{L}_{F}^{*}$-majorized mappings in $F C$-spaces, Nonlinear Anal. 72 (2010), no. 1, 65-76.

[11] P. Q. Khanh, N. H. Quan, and J. C. Yao, Generalized KKM type theorems in GFCspaces and applications, Nonlinear Anal. 71 (2009), no. 3-4, 1227-1234.

[12] S. Park, Remarks on topologies of generalized convex spaces, Nonlinear Funct. Anal. Appl. 5 (2000), no. 2, 67-79.

[13] Comments on fixed point and coincidence theorems on multimaps with nonconvex or noncompact domains, Varāhmihir J. Math. Sci. 6 (2006), no. 1, 15-24.

[14] - Various subclasses of abstract convex spaces for the KKM theory, Proc. National Inst. Math. Sci. 2 (2007), no. 2, 35-47.

[15] Elements of the KKM theory on abstract convex spaces, J. Korean Math. Soc. 45 (2008), no. 1, 1-27.

[16] Equilibrium existence theorems in KKM spaces, Nonlinear Anal. 69 (2008), $4352-4364$.

[17] New foundations of the KKM theory, J. Nonlinear Convex Anal. 9 (2008), no. $3,331-350$.

[18] _ Fixed point theory of multimaps in abstract convex uniform spaces, Nonlinear Anal. 71 (2009), no. 7-8, 2468-2480.

[19] _ Generalized convex spaces, L-spaces, and FC-spaces, J. Global Optim. 45 (2009), no. 2, 203-210.

[20] , Comments on generalized R-KKM type theorems, Comm. Korean Math. Soc. 25 (2010), no. 2, 303-311.

[21] _ The KKM principle in abstract convex spaces: equivalent formulations and applications, Nonlinear Anal. 73 (2010), no. 4, 1028-1042. 
[22] K. Włodarczyk and D. Klim, Equilibria and fixed points of set-valued maps with nonconvex and noncompact domains and ranges, Nonlinear Anal. 65 (2006), no. 4, 918-932.

The National Academy of Sciences

REPUBlic OF Korea

AND

Department of Mathematical Sciences

Seoul National University

SEOUl 151-747, Korea

E-mail address: shpark@math.snu.ac.kr 